\title{
Anaesthetic Management of Cerebral Arterio-venous Malformation Excision Using Awake Craniotomy: Initial Experience of Two Cases
}

\author{
Waleed Bin Ghaffar1, Khalid Ahsan'1, Faraz Shafiq1 and Ather Enam² \\ Department of Anaesthesiology1 / Neurosurgery22, The Aga Khan University Hospital, Karachi, Pakistan
}

\begin{abstract}
This case series summarises the successful management of two cases with cerebral arterio-venous malformation (AVM). In first patient, it was located superficially in right frontal cortex, while the second one had this in left parieto-temporal region. Both were excised successfully using awake craniotomy. The patients had their assessment and psychological preparation by neuro-anesthetist and neurosurgeon. Along with the routine monitoring, invasive arterial line, and bispectral index monitoring was used to monitor the sedative effect of propofol. Intraoperative analgesia was provided using scalp block. The anaesthetic management was helpful in facilitating intraoperative neurological monitoring using verbal and motor responses. Patients remained pain-free and hemodynamically stable during resection phase. The postoperative period showed adequate pain control, decreased postoperative nausea/vomiting and shorter length of stay in the hospital. Both patients had $100 \%$ obliteration of AVM confirmed via cerebral angiography.
\end{abstract}

Key Words: Anaesthesia, Arteriovenous malformation, Craniotomy, Wakefulness.

How to cite this article: Ghaffar WB, Ahsan K, Shafiq F, Enam A. Anaesthetic management of cerebral arterio-venous malformation excision using awake craniotomy: Initial experience of two cases. J Coll Physicians Surg Pak 2019; 29 (Supplement 2):S74-S76.

\section{INTRODUCTION}

Cerebral vascular malformations present a variety of lesions, amongst which arterio-venous malformation (AVM) is the most common. Its estimated incidence is 1:2,000-1:5,000.1 Due to decreased morbidity and low risk of recurrence, microsurgical excision remains the best approach in selected patients. ${ }^{2}$ However, excision of AVM in an eloquent area is challenging, where preservation of neurological functions becomes important. Anaesthetic management can play significant role in improving outcome of these patients by facilitating intraoperative neurological monitoring. The use of awake craniotomy (AC) in this scenario is underused. ${ }^{3-5}$ The objective of this case series is to share our experience of managing two cases of AVM excision utilising awake throughout (AT) approach. The importance of AC and its impact on intraoperative neurological monitoring, resection and outcome is highlighted in the case series.

\section{CASE REPORT}

Case 1: A 27-year female with no known comorbids, weighing $57 \mathrm{~kg}$, presented with a history of generalised

Correspondence to: Dr. Waleed Bin Ghaffar, Resident, Department of Anaesthesiology. The Aga Khan University

Hospital, Stadium Road, Karachi, Pakistan.

E-mail: waleedghaffar@hotmail.com

Received: January 09, 2019; Revised: April 08, 2019;

Accepted: April 09, 2019 headache and right-sided facial nerve palsy for three years. General and systemic examination was normal. Baseline laboratory parameters were unremarkable. MRI revealed abnormal mass of vessels having "bag of worms" appearance in right frontal lobe, with central nidus measuring $13 \times 7 \mathrm{~mm}$ in non-eloquent area (Figure 1). Spetzler Martin grade 1 was given to this malformation.

Case 2: A 20-year female with no associated comorbids, weighing $50 \mathrm{~kg}$, presented with a history of left parietotemporal headache and focal seizures for the past one month. General and systemic examination was normal. Baseline laboratory parameters were unremarkable. Her CT scan and angiogram showed left parietotemporal AVM having surrounding gliosis and intra-nidal aneurysm (Figure $2 A$ and $B$ ). It was given grade 3 on Spetzler Martin AVM grading system.

An AC was planned, for which patients had preoperative assessment and psychological preparation by neuroanesthetist. SBAR (Situation, background, assessment and recommendations) approach was used to declare patients' willingness to proceed with awake option. They were explained thoroughly regarding procedure, operating room setup and provided with education brochure to familiarise themselves with forthcoming events. Routine American Society of Anesthesiologist (ASA) standard monitoring was instituted. An invasive radial arterial line and a large bore IV cannula was also placed. Supplemental oxygen was given via nasal prong @ 2 L/min of flow. Scalp block was done using 


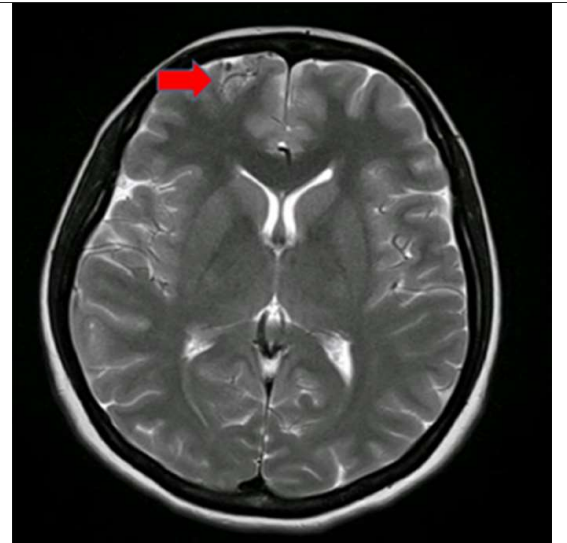

Figure 1: MRI image showing small bunch of vessels with dilated veins in right frontal lobe.

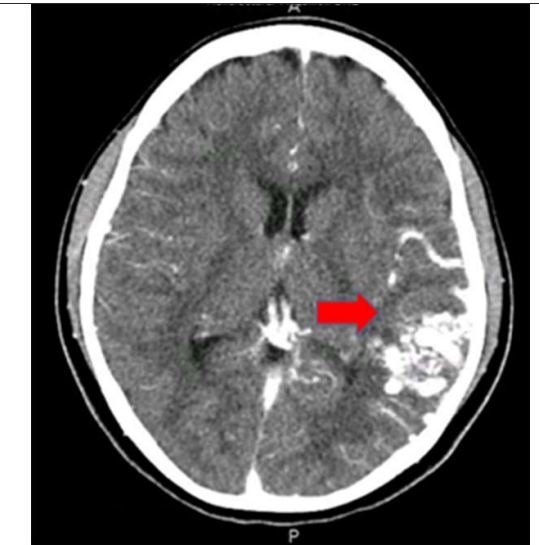

Figure 2A: Left parieto-temporal arteriovenous malformation.

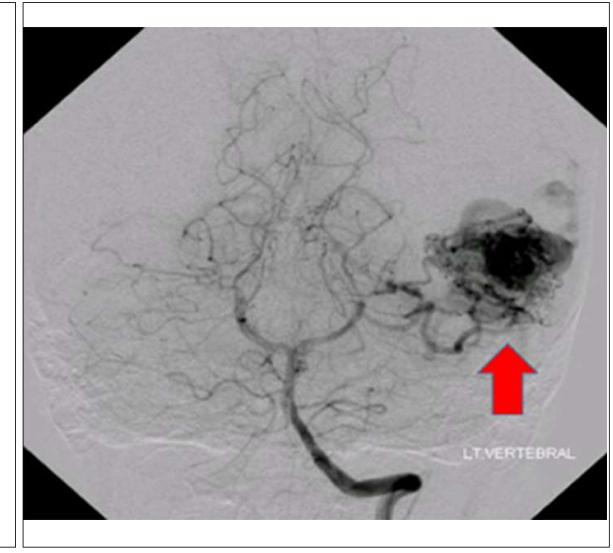

Figure 2B: AV malformation in left temporal region measuring $26 \times 14 \mathrm{~mm}$. anatomical landmark technique targeting individual nerves. ${ }^{5}$ After confirmation of block, Mayfield surgical pins were applied. Propofol infusion was used for conscious sedation at the rate of $1.5-2 \mathrm{mg} / \mathrm{kg} / \mathrm{hr}$. The level of sedation was guided by bispectral index (BIS), the target score of which was kept between 80-90. Dual antiemetic prophylaxis was provided using ondansetron and dexamethasone, $0.1 \mathrm{mg} / \mathrm{Kg}$. Antiepileptic prophylaxis was given using levitracteatum $1 \mathrm{~g}$, intravenously. Besides, ice-cold ringer lactate solution was made ready to treat any seizure activity during the procedure. Cortical stimulation was done using unipolar electrode. Intraoperative neurological functions were evaluated by monitoring verbal and motor responses. Patients remained hemodynamically stable and painfree during resection phase and throughout the procedure. Intraoperative blood loss was 150 and $350 \mathrm{ml}$ respectively, while the surgery lasted for 160 minutes in the first patient, and 270 minutes in the second. Postanesthesia care unit (PACU) stay was uneventful, and both patients were discharged to a special care unit (SCU) and to home after confirmation of cerebral angiography, which revealed no residual malformation. The postoperative analgesia was prescribed with intermittent doses of paracetamol and tramadol. The first patient was discharged at second postoperative day (POD). The second patient developed transient aphasia during postoperative stay at SCU. This was resolved completely after 48 hours, and she was discharged on $4^{\text {th }} \mathrm{POD}$.

\section{DISCUSSION}

The use of $A C$ for excision of cerebral $A V M$ is underutilised, which is evident in current literature either by isolated case reports or retrospective studies. A recent study from Hong Kong shared their retrospective data of 13 years, during which they have done six cases using AC. They shared the significance of awake approach for intraoperative neurological monitoring and its association with higher obliteration rate. Gamble et al. reported their six-year experience, where they have done 42 AVM repairs. AC was associated with better surgical cure and proven to be useful for intraoperative neurological monitoring. The use of $A C$ is recently being utilised at our healthcare set up for excision of supratentorial tumors near eloquent cortex. 5 It has been found to be useful. The extension of this technique for cases like AVM and clipping aneurysms is a step ahead toward better care and resource utilization for underdeveloped countries. Chan et al. emphasised the conduct of AVM repair under AC. They concluded that the success of procedure was dependent upon two factors, i.e. anaesthetic competence and cooperation of the patient. ${ }^{3}$ At our institute, all patients scheduled for AC are assessed by neuro-anaesthestist. This includes a review of a patient's education brochure in first instance; followed by detailed presentation depicting theatre setup, scalp block and sharing of patient experiences. As mentioned above, this is based on SBAR approach. In our first case, patient had AVM in non-eloquent area. In this patient, AC was offered because of its superficial location and secondly to provide hemodynamic stability. We know that local hemodynamic changes in brain due to AVM cause vasodilation of adjacent vessels for preservation of perfusion. After repair of low resistance AVM, adjacent vessels are exposed to higher pressures. Thus, normal perfusion pressure breakthrough may happen, as adjacent vessels are unable to autoregulate in normal pressure range due to disrupted autoregulation. This can cause hemorrhage. That is why strict blood pressure control is advised, which is really difficult to manage in patients with general anaesthesia (GA). That may be an added advantage of using AC for neurosurgical cases, where its efficacy can be predicted by low complication and failure rate in comparison to GA. ${ }^{6}$ Besides, AC is also associated with decreased incidence of postoperative nausea and vomiting, which is again beneficial to blunt hemodynamic responses in these cases. In our second case, AC was offered for intraoperative neurological monitoring, which was done 
successfully. Pre-surgical AVM embolisation was done that might lead to increased probability of complete obliteration. ${ }^{7}$ In the presence of an associated aneurysm, deep venous drainage and deeper location of AVM; the risk of subsequent hemorrhage is increased. ${ }^{7}$ To blunt the hemodynamic and stress response to Mayfield pins, AC was the best option. ${ }^{8}$

Postoperative course of these patients showed significant reduction in pain score and an overall reduction in length of stay at PACU, SCU and in the hospital. Both of which are the reported advantage of using AC. ${ }^{9}$ There are reports of neurological dysfunctions even by opting awake approach. Zhou et al., in their retrospective study were unable to find any differences in neurologic dysfunction. ${ }^{10}$ For AVM in a functional area, they reported three cases of postoperative aphasia despite normal intraoperative course. Our second patient also suffered postoperative aphasia but it was settled within two days. Gamble et al., in their case series of four patients, reported one case of postoperative dysphasia. This was recovered within 6 weeks. ${ }^{4}$ Though AC is best for resection of selected AVM cases, we need randomised controlled trials to determine its overall benefits including the neurological outcome, cost and satisfaction related to technique.

$A C$ is a suitable option for selected AVM excisions, which lie either superficially or in eloquent cortex. The approach is useful in facilitating intraoperative neurological monitoring, providing hemodynamic stability and quality analgesia. The benefit of technique needs to be explored in terms of cost including the length of stay in the hospital.

\section{PATIENTS' CONSENT:}

Written and informed consents were obtained from both the patients to publish the data.

\section{CONFLICT OF INTEREST:}

Authors declared no conflict of interest.

\section{AUTHORS' CONTRIBUTION:}

WBG: Manuscript writing, submission, proofreading.
KA: Data collection.

FS: Manuscript writing, proofreading.

AE: Patient recruitment, surgical input.

\section{REFERENCES}

1. Saleh O, Baluch A, Kaye A, Kaye A. Arteriovenous malformation, complication, and perioperative anesthetic management. Middle East J Anaesthesiol 2008; 19:737-56.

2. Schramm J, Schaller K, Esche J, Boström A. Microsurgery for cerebral arteriovenous malformations: Subgroup outcomes in a consecutive series of 288 cases. J Neurosurg 2017; 126: 1056-63.

3. Chan DYC, Chan DTM, Zhu CXL, Kan PKY, Ng AY, Hsieh Y-PS, et al. Awake craniotomy for excision of arteriovenous malformations? A qualitative comparison study with stereotactic radiosurgery. J Clin Neurosci 2018; 51:52-6.

4. Gamble AJ, Schaffer SG, Nardi DJ, Chalif DJ, Katz J, Dehdashti AR. Awake craniotomy in arteriovenous malformation surgery: the usefulness of cortical and subcortical mapping of language function in selected patients. World Neurosurg 2015; 84:1394-401.

5. Shafiq F, Salim F, Enam A, Parkash J, Faheem M. Anaesthetic management of supratentorial tumor craniotomy using awakethroughout approach. J Coll Physicians Surg Pak 2017; 27: 775.

6. Sacko O, Lauwers-Cances $V$, Brauge D, Sesay M, Brenner A, Roux F-E. Awake craniotomy vs. surgery under general anesthesia for resection of supratentorial lesions 4 . Neurosurgery 2011; 68:1192-9.

7. Bendok BR, El Tecle NE, EI Ahmadieh TY, Koht A, Gallagher TA, Carroll TJ, et al. Advances and innovations in brain arteriovenous malformation surgery. Neurosurgery 2014; 74 (Suppl 1):S60-S73.

8. Geze S, Yilmaz AA, Tuzuner F. The effect of scalp block and local infiltration on the haemodynamic and stress response to skull-pin placement for craniotomy. Eur J Anaesthesiol 2009; 26:298-303.

9. Guilfoyle MR, Helmy A, Duane D, Hutchinson PJ. Regional scalp block for postcraniotomy analgesia: A systematic review and meta-analysis. Anesth Analg 2013; 116:1093-102.

10. Zhou Q, Li M, Yi L, He B, Li X, Jiang Y. Intraoperative neuromonitoring during brain arteriovenous malformation microsurgeries and postoperative dysfunction: A retrospective follow-up study. Medicine (Baltimore) 2017; 96:e8054. 